Recibido: 20 Noviembre / Aceptación: 10 Diciembre

Reseña

\title{
¿Los resultados de investigación permean el aula de clase?
}

\author{
Martha Cecilia Mosquera Urrutia \\ Docente de planta del Programa de Licenciatura en Matemáticas \\ Universidad Surcolombiana \\ martha.mosquera@usco.edu.co
}

Durante los días 14 al 18 de Septiembre de 2015, se llevó a cabo en la Universidad Surcolombiana, el -VII FORO INTERNACIONAL DE MATEMÁTICAS (FIMUSCO), IV ENCUENTRO DE SEMILLEROS DE INVESTIGACIÓN EN MATEMÁTICA EDUCATIVA Y III ENCUENTRO DE EGRESADOS DE LOS PROGRAMAS DE LICENCIATURA EN MATEMÁTICAS Y FISICA Y MATEMÁTICAS DE LA UNIVERSIDAD SURCOLOMBIANA, Organizado Por El PROGRAMA DE LICENCIATURA EN MATEMÁTICAS Y El GRUPO E.MAT.H "Educación MATemática en el Huila" Este evento logró congregar a unos 380 participantes entre estudiantes de la universidad, egresados, docentes del Departamento, estudiantes de las universidades: Pedagógica Nacional y Externado en Bogotá; Tolima en Ibagué; Amazonia en Florencia y CORHUILA en Neiva.

El FIMUSCO es una actividad a través de la cual el programa de Licenciatura en Matemáticas y el GRUPO E.MAT.H buscan reunir a la comunidad académica en un espacio de reflexión alrededor de una pregunta, convocando para ello conferencistas de talla regional, nacional e internacional quienes aportan con su trabajo al desarrollo del evento.

En esta ocasión en torno a la pregunta sobre si: ¿los resultados de investigación permean el aula de clase? se presentaron Conferencias, Talleres, Experiencias de Aula, Ponencias, Posters y Comunicaciones Breves.

Según los especialistas, los inicios de la investigación en Didáctica de la Matemática se ubican a finales del SXIX época en la cual la formación y preparación de los profesores empezó a hacer parte de programas de educación superior. La investigación surge por la necesidad de contar con docentes altamente cualificados, con una formación profesional pertinente; la cual más allá de "tener sólidos conocimientos en el área de matemáticas", como requisito para enseñarlas, propende por un profesional que conozca la disciplina, los problemas de su enseñanza y aprendizaje y el contexto en que se realiza la enseñanza, entre otros. En la entrevista central organizada por el Centro de Producción Audiovisual de la Universidad Surcolombiana la profesora Martha Cecilia Mosquera Urrutia coordinadora del FIMUSCO, dialogó con tres de los Invitados: PhD. Ricardo Cantoral Uriza de CINVESTAVMéxico, PhD. Raimundo Ángel Olfos Ayarza de la PUCV-Chile y PhD. Bernardo Recamán Santos de la Universidad de Los Andes-Bogotá.

\section{¿Los resultados de la investigación permean el aula de clase?}

Frente a este interrogante el PhD. Ricardo Cantoral opina que es un debate de orden mundial, surgido en la posguerra para responder al Programa de una matemática para todos, pensada desde la necesidad del que aprende; en ese sentido afirma que lo que se plantea de fondo es ¿cuál es el impacto que la investigación produce? Y presenta dos ejemplos para mostrar que si hay un impacto; el primero alude al aporte de la investigación básica a la matemática educativa, en relación con la tecla para graficar que hoy tienen las calculadoras y que permite realizar varias representaciones de un objeto para poder comprenderlo y el segundo se refiere a la investigación que piensa en el profesor como una variable, pero no como el culpable de los problemas educativos, sino en el desarrollo profesional del docente y la formación del profesorado como un elemento determinante para transformar el sistema educativo.

El PhD. Bernardo Recamán afirma para el caso colombiano que el impacto de la investigación en el aula es muy bajo y muestra como evidencia el hecho de que a pesar de los avances tecnológicos y el gran auge de la matemática en el siglo XX, la matemática que se enseña hoy en día es de 1.900, muchos docentes siguen haciendo sus clases utilizando como único recurso el tablero y el marcador, hay evidencia que en América Latina los docentes son bastante dependientes de los libros de texto, en especial del Álgebra de Baldor y muchos por el estilo, hecho que les impide traer al aula problemas reales para estudiarlos. La mayoría de las investigaciones que se hacen desde el escritorio estudian la escuela, pero una vez se tienen los resultados estos no regresan a ella.

En ese orden de ideas el PhD. Raimundo Olfos opina que a pesar de haber investigaciones de hace treinta 
años o más con importantes conclusiones sobre diferentes fenómenos y problemas didácticos, es muy difícil lograr que el sistema educativo las asuma; presentando como ejemplo el avance de la tecnología, según el cual muchos conceptos que se enseñaban hace treinta años, hoy no son válidos y sin embargo en la escuela se siguen enseñando igual. Afirma que para que la investigación logre permear las aulas, es necesario que los docentes participen activamente en ella, pero a la par debe mejorarse la comunicación entre los investigadores y los docentes, sin dejar de lado aspectos determinantes como las políticas públicas, los currículos y planes de estudio, los aspectos socioculturales y las instituciones.

\section{¿Atendiendo al hecho de que ustedes además de ser investigadores son profesores, cuál es su postura desde la experiencia?}

El PhD. Raimundo Olfos comenta que cuando inició su experiencia como maestro de aula, supo que no tenía las herramientas para "enseñar la matemática", buscando respuestas hizo su magíster en psicología del aprendizaje entendiendo posteriormente que hay variables que influyen en el aprendizaje, como las relaciones interpersonales, la política y las condiciones sociales, hecho que lo llevó a emprender estudios de doctorado en sociología. Siempre ha creído que el docente debe investigar desde su práctica por ello en la actualidad su interés se centra en el estudio de clases, "El Estudio de Clases es un método japonés que consiste en planear y probar las actividades con los alumnos. Mediante conocimiento compartido (PCK) que se ha teorizado, integrado y unificado en teorías propuestas por los profesores para la enseñanza de las matemáticas..."

El PhD. Ricardo Cantoral opina que la investigación no puede hacerse, ni fuera del aula ni sin maestros, en muchos casos el profesor puede considerarse como una víctima por cuanto para realizar el trabajo requiere de condiciones, materiales, recursos y formación, porque la innovación nace del conocimiento; a manera de ejemplo refiere a un médico de provincia sin recursos y sin acceso a la información y compara su actividad con la de un médico de ciudad ubicado en un hospital de tercer nivel.

Fundador de la Teoría Socioepistemológica de la Matemática Educativa. "La Socioepistemología, como sistema teórico, se ocupa del problema que plantea la constitución del saber entre la población. Su primera contribución radica en haber producido una: descentración del objeto. Con este enfoque se asume la legitimidad de toda forma de saber, sea este popular, técnico o culto, pues en su conjunto constituyen la sabiduría humana. Plantea la educación de las matemáticas y de las ciencias desde las actividades cotidianas de las comunidades" uno de los principales logros desde su hacer lo constituye el haber logrado participar en la formulación de la política educativa, hecho que sin lugar a dudas ya muestra un progreso notorio entre la comunidad educativa mexicana, en donde se han constituido redes y comunidades de aprendizaje entre docentes e investigadores llevando a un mejoramiento progresivo de la matemática educativa en México y a un posicionamiento destacado entre la comunidad matemática internacional.

El PhD. Bernardo Recamán por su parte, indica que en América Latina los cambios se viene dando pero las reflexiones necesarias que son las que posibilitan un cambio a nivel educativo no se han dado en las esferas correspondientes, la formación inicial y continua de los docentes presenta un desfase y en la mayoría de los casos falta de pertinencia, un sistema que no es permeado por la investigación está condenado a permanecer en el pasado. En su famoso libro Póngame un Problema, plantea entre otras cosas lo siguiente: "... son muchos los profesores y estudiantes que no encuentran suficiente material atractivo en los textos escolares tradicionales, repletos de ejercicios rutinarios y tediosos, pero escasos en desafíos interesantes y otras muestras de la belleza de las matemáticas y de su carácter recreativo..."

\section{¿Y su opinión sobre los semilleros de investigación, los niños y jóvenes investigadores y la investigación escolar?}

Al respecto los investigadores coinciden en que esta iniciativa propia del sistema educativo colombiano es muy útil y permite interesantes desarrollos en la población; motiva a niños y jóvenes para acercarse a la ciencia y a la tecnología y desarrollar competencias desde temprana edad; afirman que la experiencia colombiana hoy en día se replica en varios países de América y Europa. En relación con los eventos para el intercambio de ideas y difusión de conocimientos como el FIMUSCO por ejemplo, además de felicitar la iniciativa consideran importante el posicionamiento que se logra a través de estos espacios, al permitir un diálogo directo entre los estudiantes, los profesores y los investigadores.

Links videos:

https://www.youtube.com/watch?v=I5T-j2Sd_Nw https://youtu.be/sQ6dgXmDrc8

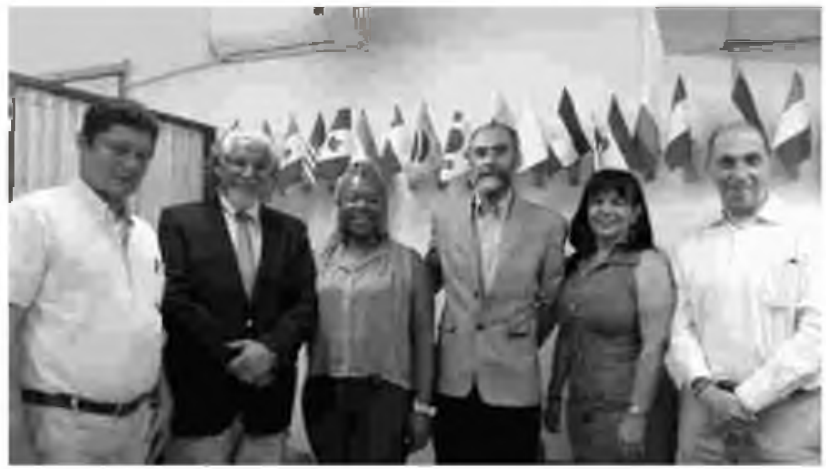

Dr. Alberto Ducuara Vicerrector administrativo USCO

PhD. Ricardo Cantoral Uriza CINVESTAV México

Dra (c) Martha Cecilia Mosquera USCO - PUCV

PhD. Raimundo Olfos Ayarza PUCV Chile

Dra. Gloria Cotrino Trujillo ORNI - USCO

PhD. Bernardo Recamán Santos UNIVERSIDAD DE LOS ANDES, Bogotá. 